\title{
EVALUATION OF GRAPHIC GESTURE IN PARKINSON'S Disease
}

\author{
VimerCATI,S.; GALli, M.; DE PANDIS, M. F.; \\ Cimolin, V.\& Albertini, G.
}

Abstract: The quantitative analysis of graphic gesture in subjects with Parkinson's Disease (PD) was carried out with an approach based on optoelectronic systems. Graphic motor deficits are in fact strongly representative and early detectors of PD. PD subjects were asked to hand draw a spiral while in on and off medication conditions, in order to highlight the effect of levodopa treatment on the graphic performance. Since cognitive decay and dementia often occur with the developing disease the cognitive capabilities were assessed in subjects with $P D$ and subjects with PD and dementia (PDD) by administering the clock drawing test. Markers were put on the pen and sheet and the gesture was acquired with an optoelectronic system. This system was selected as it is suitable for 3D, quantitative and multifactor evaluation of the gesture. The results highlighted the validity of graphic tests in the assessment of both motor and cognitive deficits caused by PD and, moreover, uncovered the possibility to use a new quantitative, not invasive, $3 D$ method for quantifying the kinematic aspects of the graphic gesture. Further research is focussing on the assessment of both graphic and postural aspects during writing and drawing.

Key words: Parkinson's Disease, motor, cognitive, optoelectronic, graphic, drawing
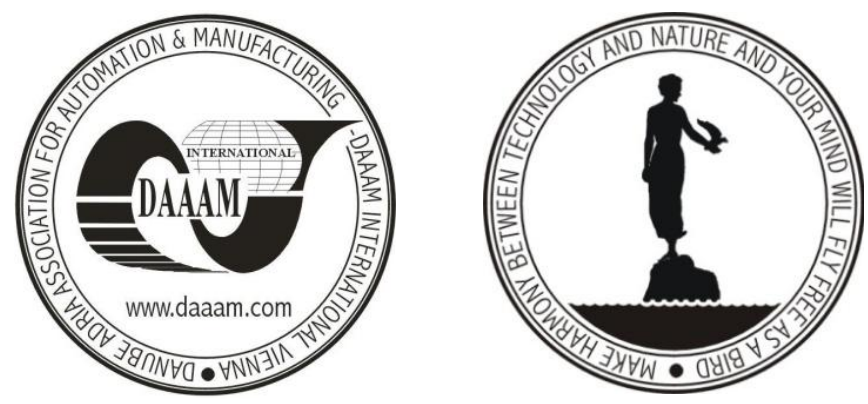

Authors' data:Eng. Vimercati, S[ara]*; Prof. Galli, M[anuela]*; **MD De Pandis M[aria] F[rancesca]***; PhD Cimolin V[eronica]*; MD Albertini G[iorgio]**,*Bioeng. Dept, Politecnico di Milano, P.zza Leonardo da Vinci 32, Milan, Italy, **IRCCS "San Raffaele Pisana", Roma, Italy, ***San Raffaele Cassino Institute, Cassino (FR), Italy. vimercati.sara@gmail.com, manuela.galli@polimi.it, maria.depandis@sanraffaele.it, veronica.cimolin@polimi.it, giorgio.albertini@sanraffaele.it

This Publication has to be referred as: Vimercati, S[ara]; Galli, M[anuela]; De Pandis, M[aria F[rancesca]; Cimolin, V[eronica] \& Albertini, G[iorgio] (2010). Evaluation of Graphic Gesture in Parkinson's disease, Chapter 31 in DAAAM International Scientific Book 2010, pp. 321-336, B. Katalinic (Ed.), Published by DAAAM International, ISBN 978-3-901509-74-2, ISSN 1726-9687, Vienna, Austria

DOI: $10.2507 /$ daaam.scibook.2010.31 
Vimercati, S.; Galli, M.; De Pandis, M. F.; Cimolin, V. \& Albertini, G.: Evaluation...

\section{Introduction}

Writing and drawing are the final output of a complex neurological, psychological and motor action; motor and/or cognitive diseases leave therefore important traces in the graphic gesture: when a cerebral area is damaged the graphic ability is damaged too, in a way that can be related to the injured function.

Through the analysis of some aspects of the graphic gesture it is therefore possible to obtain a report of the subject's psychophysical health. Scientific interest for writing and drawing tests has been growing during the last decade because of their being non-invasive, reliable and suitable for the analysis of different pathologies, besides being well tolerated by pathological patients. In addition, writing and drawing tests can be used to investigate both the motor capabilities and the cognitive functions of the subjects.

\subsection{Assessment of motor capabilities}

In the graphical deficits due to motor difficulties it can be found the presence of diseases such as Parkinson's Disease (PD) (Lange, 2006; Tucha, 2006; Longstaff, 2003); PD symptoms include bradykinesia, tremor, rigidity, reduced manual ability and loss of accuracy, which cause typical phenomena that can be classified using graphic gesture analysis and then related to the specific pathology (Tucha, 2006).

In literature there are several studies finalized to the quantification of kinematic alterations in the graphic gesture in patients with PD. These studies demonstrate that the pathology causes a worsening in the graphic movement execution and therefore PD patients are impaired in the execution of the automatic movements of writing and drawing (Caligiuri et al., 2006; Lange et al., 2006; Tucha et al., 2006).

Spirography is a standard method to test the presence of PD symptoms, such as micrographia or tremor, or to study the strategies adopted by the patients in order to partially counter their deficit (Saunders-Pullman et al., 2008; Longstaff et al, 2006; Longstaff et al., 2003). In particular, hand-drawn spirals allow the study of micrographia and of the motor strategies adopted by the subjects [5, 7-8], because the subjects can freely choose the dimensions of the spiral and their drawing velocity. Several studies, in fact, observed that during a spiral hand-drawing task the spiral radius was shorter for the PD subjects than for the healthy subjects, leading to spirals of smaller dimensions (Saunders-Pullman et al., 2008).

A very recent work (Stanley et al., 2010) also suggests that spiral analysis may be more sensitive in detecting early changes in motor performance than the UPDRS, which is a clinical standard method for PD evaluation. The study shows that spiral execution abnormalities may have the potential to discern pre-clinical disease, and might therefore be considered in a battery of screening tests for at-risk individuals.

In some studies concerning spiral drawing the subjects are not allowed to rest the forearm on the table, so that movements can be of larger amplitude. In this way both distal and proximal joints are involved and it is therefore possible to globally study the movement of the upper limb (Pullman, 1998; Balàs et al., 2006). 
Actually the evaluation of drawing and writing tests is based on the subjective evaluation of the written paragraphs/words and drawings produced by the patients under specific requests. Only some studies investigate the graphic ability of subjects using the digitising tablets, a sort of "computerized blackboards" on which the subjects write, and which detect pen pressures (2D analysis) (Stanley et al., 2010; Pullman, 1998). Unfortunately this kind of systems is sometimes not welcome by the evaluators and by the tested patients for the unnatural condition related to their use. The tablets require the use of a specific pen and do not allow to position and rotate the paper according to the patients' need. Besides this, no information about the postural behaviour (position of head, trunk, upper limbs...) during the writing and drawing tests is provided.

Since graphic measurements are an useful instrument for the diagnosis of fine motor control problems, for the follow-up of the performance with developing disease, and also for the assessment of the pharmacological treatment effects, it can be seen the importance of having an easy, not invasive and standard experimental set-up for the acquisition of writing and drawing, which offers the possibility to threedimensionally analyze the kinematic of drawing and writing movements, as well as the related postural problems; we therefore developed a preliminary method for the acquisition and quantitative analysis of graphic gesture in PD subjects using an optoelectronic system.

\subsection{Assessment of cognitive capabilities}

Cognitive capabilities are generally evaluated in clinics through the use of simple drawing tests, for instance to assess the presence of dementia in the elderly (Mini Mental State Evaluation, Clock Drawing Test) or the presence of developmental problems, such as dyslexia and dysgraphia, in children (Denver Developmental Screening Test). In the cognitive deficits of the elderly it can be found the presence of different typologies of dementia (e.g. Alzheimer's disease AD, Mild Cognitive Impairment MCI, Parkinson's disease dementia PDD), which affect specific areas of cognition. Through the use of focused drawing tests it is possible to separately investigate the function of the different cerebral areas of cognition: this allows having an insight on how the dementia is developing and which areas have already been affected [4-7]. The Clock Drawing Test (CDT) is a qualitative neurological drawing test commonly used as a screening instrument for cognitive capabilities in the senile population and to evaluate the functional capabilities in the elderly. The test is easily administrable, requires little time and has a good sensitivity in measuring the cognitive functions in the elderly.

The CDT can be administered in two modalities: verbal command (command condition) and copying (copy condition).

In the first one (command condition) the subject is asked to draw a clock with the clock hands indicating a particular time ("ten minutes past eleven") (Hubbard \& Santini, 2008; Shah, 2002). A well-drawn clock is supposed to have the circle, numbers from 1 to 12 in the correct order and position inside the circle and the hands on the correct time. It is also possible to give the subject a pre-drawn circle, in order 
to avoid that a badly-drawn circle, or a too small one, influences the rest of the drawing (Chiu \& Li, 2008; Seber et al., 2008; Shah, 2002). The CDT command condition investigates the subject's language function (verbal comprehension); memory functions (recall of a visual engram, short-term storage, recall of time setting instructions) and executive function. The test is highly sensitive for temporal lobe dysfunction (due to its heavy involvement in both memory and language processes), and frontal lobe dysfunction (due to its mediation of executive planning) (Shah, 2002).

In the second modality (copy condition) the subject is given a printed clock, with the hands reading a certain time, and he is asked to replicate the drawing sideways, as accurately as possible. This modality requires less use of language and memory functions, but requires greater reliance on visuospatial and perceptual processes. The copy condition is good for assessing parietal lobe lesions (Shah, 2002); this region is responsible for stimuli recognition and for the recall of forms and structures.

The CDT is commonly administered with the "pen and sheet" modality, with no use of computerized systems of acquisition, and the evaluation and scoring is visually done by the clinician. It is clear that this method involves great limitations in terms of measurement: first of all, a visual assessment is not completely inter- and intra-rater reliable, because it is affected by the evaluator's experience and subjectivity. Secondly, it does not allow memorizing the temporal sequence of the graphic signs on the sheet. All information about the temporal evolution of the graphic signs, such as reaction time and duration, is therefore lost, with a loss of important quantitative information. The most of the cognitive assessment is therefore still based on visual scoring systems, such as the ones described for the CDT (i.e. Mendez, Roleau scoring systems) or the widely used Mini Mental State Evaluation (MMSE). No previous experiences about the evaluation of CDT with digitized tablets or other quantitative methods are reported in literature. In order to better quantify the gesture in the realization of CDT, we developed a preliminary method for the acquisition and analysis of cognitive drawing tests which allows evaluating both the qualitative and quantitative features of the drawing by means of an optoelectronic system of motion capture.

In conclusion, no previous studies applied the standard methods of quantitative motion analysis to investigate and characterize the writing and drawing ability in the different stages of the pathology and in the pre and post treatment conditions. The objective of the study was therefore to evaluate the graphic gesture of subjects with PD and subjects with dementia using the methods and equipments usually applied for quantitative movement evaluation. For both motor tests (spiral drawing) and cognitive tests (CDT) we defined and calculated some parameters of interest, which could help quantifying the motor and cognitive abilities of the subjects, and then developed a dedicated clinical report. 


\section{Materials and methods}

The control group was composed of eleven healthy $(\mathrm{H})$ subjects (Table 1, mean age: $63.7 \pm 9.4$ years old), whose ages matched the ages of the PD subjects. The $\mathrm{H}$ subjects were administered both the spiral drawing test (motor capabilities assessment) and the clock drawing test (cognitive capabilities assessment).

\begin{tabular}{|c|c|c|c|}
\hline Code & State & Gender & Age (years) \\
\hline H_1 & H & M & 67 \\
\hline H_2 & H & F & 68 \\
\hline H_3 & H & M & 73 \\
\hline H_4 & H & F & 62 \\
\hline H_5 & H & F & 71 \\
\hline H_6 & H & M & 70 \\
\hline H_7 & H & F & 68 \\
\hline H_8 & H & F & 69 \\
\hline H_9 & H & F & 59 \\
\hline H_10 & H & F & 42 \\
\hline H_11 & H & M & 52 \\
\hline
\end{tabular}

Tab. 1. Characteristics of the healthy subjects $(\mathrm{H})$ who took part to both spiral and clock drawing tests

\subsection{Assessment of motor capabilities- spiral drawing test}

The aim was to assess the spiral drawing capabilities of PD subjects and the effect of levodopa treatment on micrographia and bradykinesia. The group of Parkinson's Disease subjects (PD) was composed of fifteen subjects (Table 2, mean age: $66.8 \pm 9.8$ years old).

\begin{tabular}{|c|c|c|c|c|c|}
\hline Code & State & Gender & Age (years) & UPDRS OFF & UPDRS ON \\
\hline PD_1 & PD & F & 70 & 63 & 30 \\
\hline PD_2 & PD & F & 63 & 64 & 36 \\
\hline PD_3 & PD & M & 76 & 50 & 22 \\
\hline PD_4 & PD & F & 63 & 78 & 21 \\
\hline PD_5 & PD & F & 65 & 55 & 34 \\
\hline PD_6 & PD & M & 75 & 69 & 32 \\
\hline PD_7 & PD & M & 70 & 53 & 25 \\
\hline PD_8 & PD & F & 61 & 69 & 36 \\
\hline PD_9 & PD & F & 79 & 65 & 44 \\
\hline PD_10 & PD & F & 62 & 45 & 17 \\
\hline PD_11 & PD & F & 76 & 54 & 36 \\
\hline PD_12 & PD & F & 68 & 40 & 25 \\
\hline PD_13 & PD & F & 77 & 74 & 49 \\
\hline PD_14 & PD & F & 43 & 21 & 48 \\
\hline PD_15 & PD & M & 54 & 42 & 76 \\
\hline
\end{tabular}

Tab. 2. Characteristics of the PD subjects who took part to the spiral drawing test 
Vimercati, S.; Galli, M.; De Pandis, M. F.; Cimolin, V. \& Albertini, G.: Evaluation...

PD subjects were assessed in absence of pharmacological medication (OFF) and after the pharmacological treatment with levodopa $(\mathrm{ON})$.

The characteristics of the subjects who took part to the study are summarized in table 1. For every PD subject the scores of the Unified Parkinson's Disease Rating Scale (UPDRS- III) are detailed. The UPDRS III evaluation rates motor manifestations from 0 to 108, with 108 representing the higher deficit.

\subsection{Assessment of cognitive capabilities - clock drawing test (CDT)}

The aim was to assess the clock drawing capabilities of PD subjects with dementia, with respect to PD subjects without dementia.

Nine subjects with Parkinson's disease (PD, mean age $=73.2 \pm 7.2$ years) and nine subjects with Parkinson's disease and dementia (PDD, mean age $=64.8 \pm 6.9$ years) were evaluated. Data related to PD and PDD subjects are reported in Table 3.

\begin{tabular}{|c|c|c|c|c|c|}
\hline I.D. & State & Gender & Age (years) & $\begin{array}{c}\text { UPDRS } \\
\text { ON }\end{array}$ & MMSE \\
\hline PD_16 & PDD & F & 77 & 49 & 16.7 \\
\hline PD_17 & PDD & M & 75 & 32 & 20.0 \\
\hline PD_18 & PDD & F & 84 & 36 & 23.4 \\
\hline PD_19 & PDD & F & 81 & 44 & 22.5 \\
\hline PD_20 & PDD & F & 62 & 37 & 18.3 \\
\hline PD_21 & PDD & F & 66 & 20 & 18.2 \\
\hline PD_22 & PDD & M & 70 & 25 & 21.7 \\
\hline PD_23 & PDD & M & 76 & 36 & 22.7 \\
\hline PD_24 & PDD & F & 68 & 25 & 21.4 \\
\hline PD_25 & PD & M & 76 & 22 & 27.0 \\
\hline PD_26 & PD & F & 63 & 21 & 29.0 \\
\hline PD_27 & PD & F & 65 & 34 & 26.2 \\
\hline PD_28 & PD & M & 54 & 42 & 26.7 \\
\hline PD_29 & PD & M & 57 & 26 & 28.7 \\
\hline PD_30 & PD & F & 67 & 36 & 25.2 \\
\hline PD_31 & PD & F & 61 & 36 & 28.5 \\
\hline PD_32 & PD & F & 71 & 30 & 27.3 \\
\hline PD_33 & PD & F & 69 & 47 & 26.0 \\
\hline
\end{tabular}

Tab. 3. Characteristics of the PD and PDD subjects who took part to the CDT

For every subject the scores of the Unified Parkinson's Disease Rating Scale (UPDRS- III) and of the Mini Mental State Examination (MMSE) are detailed. According to the clinical MMSE scale a score inferior to 24/30 reveals the presence of dementia.

\subsection{Instrumental set-up}

The graphic gesture was acquired with an optoelectronic system with six cameras (SMART-D, BTS, Italy), at a frequency of $120 \mathrm{~Hz}$, and with an integrated 326 
video system (Vixta, BTS, Italy) for video recording; the acquisition was obtained by the measure of the $\mathrm{x}, \mathrm{y}$ and $\mathrm{z}$ coordinates of passive markers of diameter $=5 \mathrm{~mm}$.

For this study a preliminary protocol was used, which does not comprehend the evaluation of posture.

The first configuration (fig.1a) was used for a static acquisition, in which the subject did not take part: the pen laid on the table and the markers were acquired for five seconds, in order to calculate the position of the pen tip and allow the calculation of its position during the dynamic acquisition, in which the graphic test was executed by the subject.

In the dynamic acquisition markers were positioned both on the sheet and on the pen (fig.1b): for the dynamic acquisition the marker on the pen tip was removed, and two markers were positioned on the sheet in order to give a reference system that was fixed with the sheet.

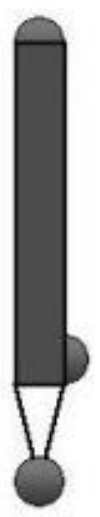

Fig.la
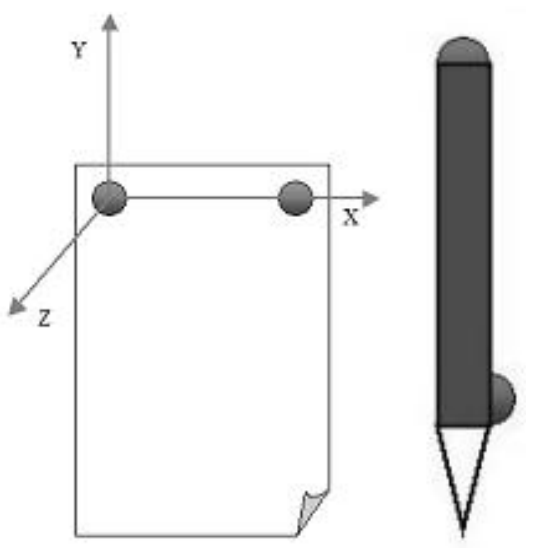

Fig.1b

Fig. 1. Configuration of the markers for the static acquisition (a) and dynamic acquisition (b)

Data reconstruction was carried out using the software Smart Tracking (BTS, Italy), which computes the tracking phase. After the tracking the acquisitions were computed using Smart Analyzer software (BTS, Italy, Version 1.10), which creates procedures ad hoc for the extraction of spatiotemporal parameters and trajectories. With this software an algorithm was defined for the automatic calculation of the parameters of interest and for the realization of the related report.

\subsection{Spiral test acquisition and parameters calculation}

The subjects were asked to hand draw a spiral, starting from the centre and with at least five revolutions (Saunders-Pullman et al., 2008; Balàs et al., 2006). During the execution the subjects were not allowed to rest their forearm on the table, so that movements could be of larger amplitude (Stanley et al., 2010; Ondo et al., 2005).

The spatiotemporal characteristics of the spiral drawing were considered, and in particular dimension of the spirals "D" $\left[\mathrm{mm}^{2}\right]$ and velocity profiles $[\mathrm{mm} / \mathrm{s}]$. Dimension (D) was calculated as an indicator of the presence of 
Vimercati, S.; Galli, M.; De Pandis, M. F.; Cimolin, V. \& Albertini, G.: Evaluation...

micrographia, and computed as the product between two perpendicular diameters of the spiral (1):

$$
\mathrm{D}=\mathrm{d}_{1} * \mathrm{~d}_{2}
$$

The velocity profile patterns of the tip of the pen were considered as a detector of the slow movements (bradykinesia) typical of PD. During the test there were no temporal limits of execution, so that the velocity could be freely chosen by the subjects.

Starting from the 3D coordinates of the markers on the pen, the pen tip coordinates were calculated, and therefore instant velocity $\mathrm{V}_{\text {ist }}$ was computed as (2):

$$
v_{i s t}=\sqrt{v_{x}^{2}+v_{y}^{2}}
$$

where $\mathrm{V}_{\mathrm{x}}$ and $\mathrm{V}_{\mathrm{y}}$ are the velocity components along the $\mathrm{x}$ and $\mathrm{y}$ directions respectively.

The velocity data were normalized with respect to time, so that velocity profiles could be plotted against a percentage of the trial duration.

A punctual mean was calculated, separately, for the velocity profiles of $\mathrm{H}, \mathrm{PDON}$ and PD OFF groups of subjects.

Linear regression was computed for the punctual mean velocity profiles, in order to describe the trends of the velocity profiles, from centre to external spires, for the three groups of subjects.

\subsection{Clock Drawing Test (CDT) acquisition and parameters calculation}

The CDT was composed of two trials. In the first one (command condition) the subject was verbally asked to "draw a clock inside the pre-drawn circle and then draw the clock hands to indicate ten minutes past eleven". In the second task (copy condition) the subject was asked to copy, inside a printed circle, the clock shown on the paper.

Figure 2 shows an example of the clock drawing trace drawn by a subject with PD and dementia in the command condition (on the left) and in the copy condition (on the right).
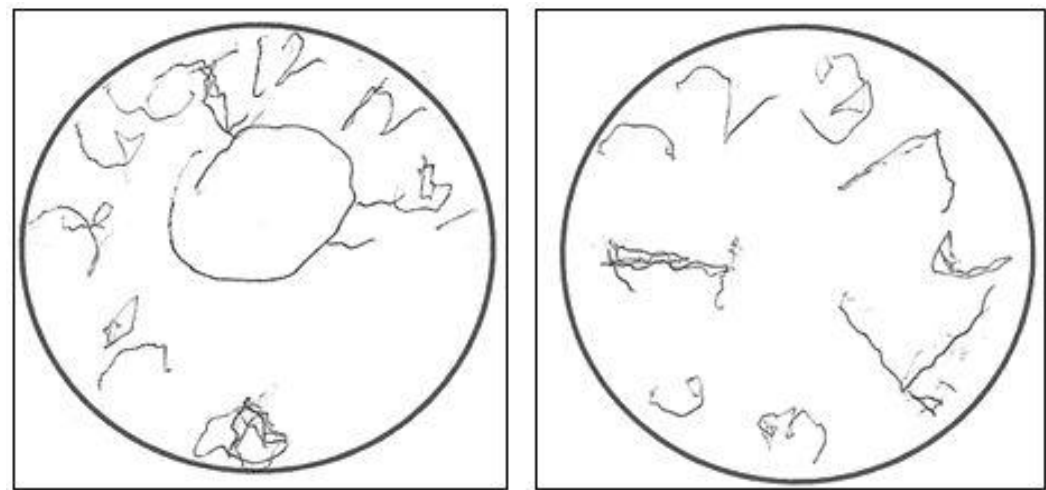

Fig. 2. Drawing trace of a clock performed by a PDD subject in the command (on the left) and in the copy condition (on the right) 
The Trial duration, TD, [s] of the trial was computed using Smart Analyzer software, in order to assess the reasoning and decision-taking time of PD and PDD subjects.

\subsection{Statistical analysis}

Data were grouped by categories, and for each group the mean and standard deviations was calculated.

For the spiral test the subjects were divided into healthy subjects $(\mathrm{H})$ and subjects with Parkinson's Disease in the on medication condition (PDON) and in the offmedication condition (PD OFF).

For the CDT the subjects were divided into healthy $(\mathrm{H})$, subjects with Parkinson's Disease (PD) and subjects with Parkinson's Disease and Dementia (PDD). PD and PDD subjects were both evaluated in the on-medication phase.

In order to give statistical validity to the parameters some statistic tests were done with Statistica software (Version 7.0). For every test the p-value was taken into account, its significance threshold being 0.05 , so that values minor than the threshold were considered statistically significant.

First, a normality test (Lilliefors and Kolmogorov-Smirnov tests) was used to verify the kind of distribution of the parameters; because of the normality tests' results nonparametric statistical tests were chosen for further analysis.

Correlation was tested by Spearman's test, while for the analysis of respectively independent variables (i.e. PD vs. PDD) and dependent variables (i.e. PDON vs. PD OFF) the Mann-Whitney U-test and the Wilcoxon Test were chosen.

\section{Results}

\subsection{Spiral test}

The results of the UPDRS III evaluation and dimensional parameter are summarized in Table 4.

\begin{tabular}{|c|c|c|c|c|c|c|c|}
\hline Code & State & Gender & Age & $\begin{array}{c}\text { UPDRS } \\
\text { OFF }\end{array}$ & $\begin{array}{c}\text { UPDRS } \\
\text { ON }\end{array}$ & $\begin{array}{c}\text { Dimension } \\
\text { OFF }\left[\mathbf{m m}^{2}\right]\end{array}$ & $\begin{array}{c}\text { Dimension } \\
\text { ON [mm }\end{array}$ \\
\hline PD_1 & PD & F & 70 & 63 & 30 & 10.7 & 19.7 \\
\hline PD_2 & PD & F & 63 & 64 & 36 & 3.0 & 5.0 \\
\hline PD_3 & PD & M & 76 & 50 & 22 & 12.9 & 11.9 \\
\hline PD_4 & PD & F & 63 & 78 & 21 & 7.3 & 9.8 \\
\hline PD_5 & PD & F & 65 & 54 & 49 & 19.1 & 10.3 \\
\hline PD_6 & PD & M & 75 & 69 & 32 & 4.9 & 6.7 \\
\hline PD_7 & PD & M & 70 & 53 & 25 & 14.6 & 27.3 \\
\hline PD_8 & PD & F & 61 & 69 & 36 & 12.9 & 8.4 \\
\hline PD_9 & PD & F & 79 & 65 & 44 & 8.5 & 12.7 \\
\hline PD_10 & PD & F & 62 & 45 & 17 & 3.7 & 12.8 \\
\hline PD_11 & PD & F & 76 & 54 & 36 & 8.8 & 9.4 \\
\hline PD_12 & PD & F & 68 & 40 & 25 & 13.5 & 12.7 \\
\hline PD_13 & PD & F & 77 & 62 & 49 & 12.7 & 12.8 \\
\hline
\end{tabular}


Vimercati, S.; Galli, M.; De Pandis, M. F.; Cimolin, V. \& Albertini, G.: Evaluation...

\begin{tabular}{|l|l|l|l|l|l|l|l|}
\hline PD_14 & PD & F & 43 & 48 & 21 & 2.2 & 2.2 \\
\hline PD_15 & PD & M & 54 & 76 & 42 & 3.9 & 5.6 \\
\hline
\end{tabular}

Tab. 4. Characteristics of the Parkinson's Disease subjects, UPDRS, dimension parameter

In Table 5 the mean values (st. dev.) of dimension are shown, and the statistical analysis is reported as well. Hand-drawn spirals analysis showed that healthy subjects globally drew spirals of bigger dimension: this means that the movement involved both the distal joint of the wrist and the proximal joint of the shoulder.

\begin{tabular}{|l|c|c|c|l|}
\hline & H & \multicolumn{2}{|c|}{ PD } & p $<\mathbf{0 . 0 5}$ \\
\cline { 3 - 5 } & & OFF & ON & \\
\hline Dimension $\left[\mathbf{m m}^{2}\right]$ & $14.80(7.72)$ & $9.24(5.04)$ & $11.15(6.13)$ & $*$ \\
\hline
\end{tabular}

Tab. 5. Mean values (st. dev.) of dimension. " $* *=p<0.05$ PD OFF vs. PD ON;

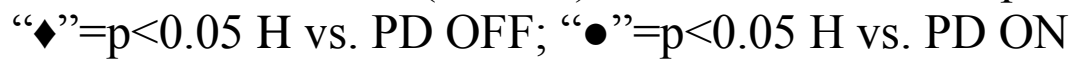

Statistical analysis showed that PD OFF subjects drew smaller spirals, so that the shoulder joint was as less involved as possible, and motor control could therefore increase (Longstaff \& Heath, 2006; Longstaff et al., 2003).

Significant differences were found between the spiral dimensions in the OFF vs. ON condition (table 5): levodopa decreases the entity of tremor and bradykinesia and, as a result, leads to better motor control and thus larger movements. No significant difference was found between PDON and $\mathrm{H}$ subjects for the dimensional parameter. As it can be noticed the most of the subjects draws spirals of bigger dimensions in the on condition, with the exception of two subjects (PD_5 and PD_8), who decreased the dimension in the on condition, and other three subjects (PD_3, PD_13 and PD_14) draw at the same dimension despite the two different pharmacological conditions, so the dimensional parameter was able to uncover micrographia in the $67.7 \%$ of the subjects.

Figure 3 shows the drawing trace of the spiral drawn by an healthy subject and the traces of two spirals, drawn by the same PD subject (PD_9), respectively in the off condition (in the centre) and in the on condition (on the right).
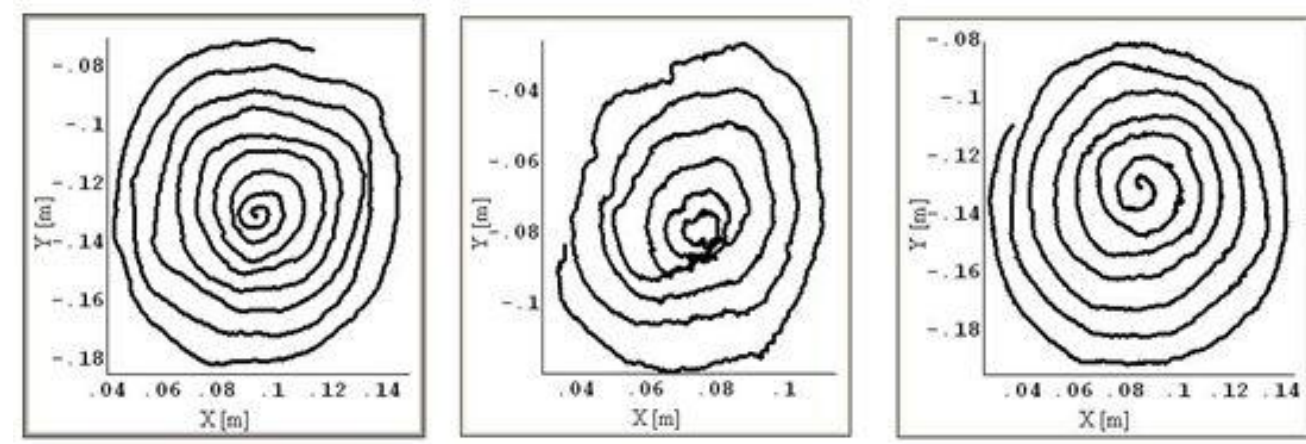

Fig. 3. Drawing trace of the spiral drawn by an healthy subject (on the left), and by the same subject with PD (PD_9), respectively in the off (in the centre) and in the on (on the right) conditions 
As it can be seen by a first visual evaluation of the drawing traces and of the scales of measurement, the spirals drawn by the subject with PD in the two conditions are different: a marked presence of tremor and irregularity is present in the off condition, while a better spatial organization and regularity in spiral increments can be found in the on condition, in which the spiral becomes very similar to the one drawn by the healthy subject. The spiral dimension is similar for the $\mathrm{H}$ and the subject with PD in the on condition, while the spiral is smaller for the PD in the off condition, as demonstrated by the $\mathrm{x}$ and $\mathrm{y}$ coordinate values.

In figure 4 the punctual mean velocities of the three groups of subjects are shown.Linear regression is plotted as well, with the line equation and the Pearson's Coefficient $\left(\mathrm{R}^{2}\right)$, which is a measure of the quality of fitting. It can be noticed that $\mathrm{H}$ subjects (black line) developed higher drawing velocities, with an increasing profile from the inside to the outside of the spiral.

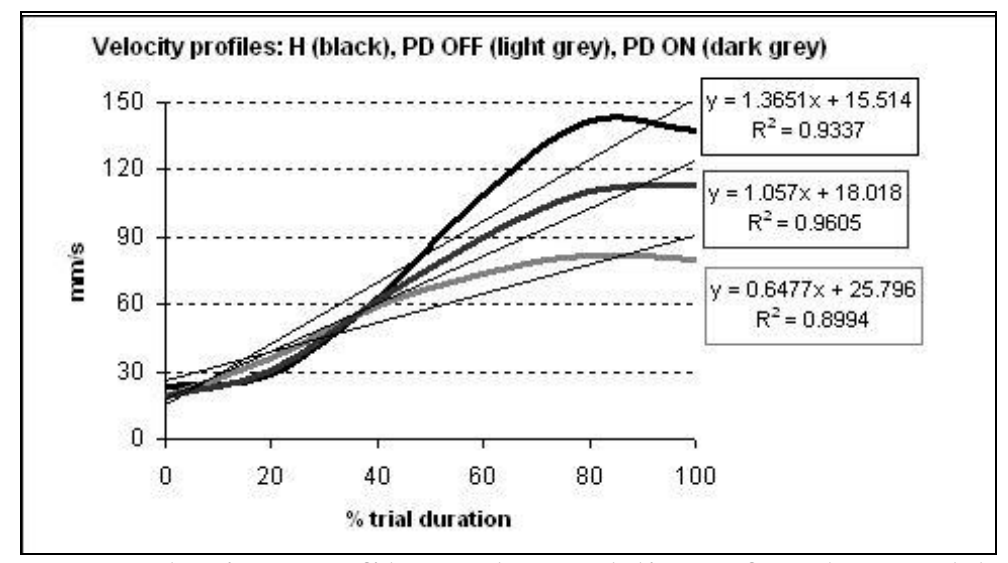

Fig. 4. Punctual mean velocity profile and trend lines for the H (black), PDON (dark grey) and PD OFF (light grey) groups, plotted against the percentage of duration of the trial

In Table 6 the angular coefficients of the three regression lines, calculated from the velocity profiles of figure 4 , are shown for comparison.

As it can be seen by Figure 5 and Table 6 the velocity range was higher for $\mathrm{H}$ subjects, who incremented their drawing velocity from inside to outside, with a trend that could be well-fitted $\left(\mathrm{R}^{2}=0.9337\right)$ by a linear regression, and an angular coefficient of the regression line of 1.365 .

Velocity ranges were significantly different between PDON and PD OFF subjects.

PD subjects, in fact, drew slower; the administration of levodopa appeared to increase the PD subjects' velocity, which in fact did not show significant differences in the $\mathrm{ON}$ condition if compared to the $\mathrm{H}$ group. As it can be observed in Table 6 the angular coefficients in the ON and OFF condition did not reach the value of the $\mathrm{H}$ angular coefficient.

\begin{tabular}{|c|c|c|c|c|}
\hline & H & & & $p<0.05$ \\
\hline & & OFF & $\mathbf{O N}$ & \\
\hline Angular coefficient of linear regression & 1.365 & 0.648 & 1.057 & $*$ \\
\hline Velocity range $[\mathrm{mm} / \mathrm{s}]$ & 118.95 & 62.67 & 94.43 & $* \bullet$ \\
\hline
\end{tabular}

Tab. 6. Angular coefficient values of the regression lines of the velocity profiles.

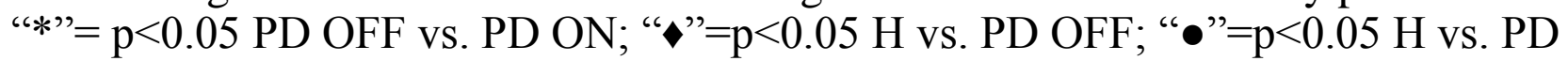
$\mathrm{ON}$ 
Vimercati, S.; Galli, M.; De Pandis, M. F.; Cimolin, V. \& Albertini, G.: Evaluation...

Comparing the two conditions, the lower angular coefficient (approximately half value) was calculated for the PD OFF group. PD OFF subjects, due to bradykinesia and fatigue, show therefore much more difficulty in increasing the velocity between subsequent spires, with much narrower ranges of velocity.

As a result, PD OFF subjects are slower in the execution of the motor task. The administration of levodopa affects positively the motor performance, even though the angular coefficient of PDON subjects does not reach the values of the $\mathrm{H}$ groups', but is set at an intermediate value between $\mathrm{H}$ and PD OFF' values.

\subsection{Clock Drawing Test}

Statistical analysis of the duration index revealed differences between PD and PDD subjects for both the command and the copy condition. The means and standard deviations of trial duration, for the two trials and the three groups, are detailed in table 7 .

\begin{tabular}{|c|c|c|c|c|c|c|}
\hline \multirow[t]{2}{*}{ TD $[\mathrm{s}]$} & \multicolumn{3}{|c|}{ CDT Command } & \multicolumn{3}{|c|}{ CDT Copy } \\
\hline & Mean & St Dev & $p<0.05$ & Mean & St Dev & $p<0.05$ \\
\hline $\mathbf{H}$ & 65.6 & 26.4 & \multirow[b]{3}{*}{$\nabla$} & 41.5 & 12.7 & \multirow{3}{*}{$\nabla$} \\
\hline PD & 72.8 & 36.1 & & 52.8 & 19.8 & \\
\hline PDD & 154.4 & 58.1 & & 104.1 & 43.4 & \\
\hline
\end{tabular}

Tab. 7. Trial duration (mean and standard deviation) for the three groups and for both drawing conditions. $*=p$-value $<0.05$ for PD vs. PDD; $*$ p-value $<0.05$ for $\mathrm{H}$ vs. PDD; $\bullet=\mathrm{p}$-value $<0.05$ for $\mathrm{H}$ vs. PD

The $\mathrm{H}$ subjects drew with comparable durations for the two tasks. No statistically significant difference was found, for duration, between H and PD subjects, while there was difference between H and PDD and between PD and PDD.

Performances were slower for both PD and PDD groups in the command condition, which therefore required more reasoning. It can be noticed that, for both command and copy condition, PD draw faster than PDD.

In order to evaluate the validity of the trial duration (TD index) in the assessment of cognitive function we calculated the correlation between MMSE and duration for the whole PD and PDD subjects, and found a that a statistical significance was present between MMSE and the two drawing conditions (Figure 5).
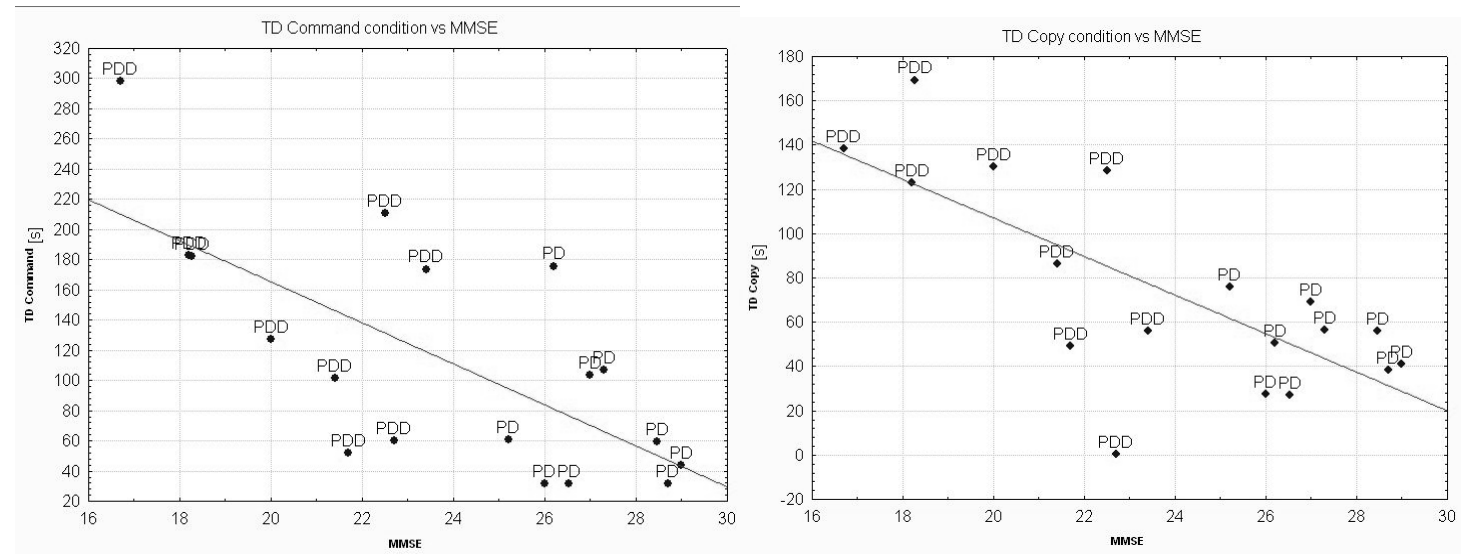

Fig. 5. Scatter plot of TD Command condition vs. MMSE (on the left) and scatter plot of TD Copy condition vs. MMSE (on the right) 
As it is shown in figure 6 anegative correlation existed between the MMSE scoring and TD for both drawing conditions, meaning that a lower MMSE (therefore a higher degree of cognitive dysfunction) led to higher reasoning time and thus to higher duration of the trials.

\section{Conclusions}

\subsection{Spiral drawing}

In this study an experimental set-up was defined for the quantitative assessment of writing and drawing. This set up was applied to a group of healthy subjects, in order to define a group of reference, and to a group of subjects with Parkinson's Disease, in order to assess the alterations caused by this pathology and to quantify the effect of levodopa treatment. The subjects with PD were tested while following withdrawal of levodopa therapy (OFF) and in the on medication condition $(\mathrm{ON})$, in order to highlight the differences caused by the pharmacological treatment on the drawing task.

In hand-drawn spirals statistical analysis showed that $\mathrm{H}$ subjects drew larger spirals, while PD OFF subjects drew smaller spirals, owing to the presence of micrographia, and according to what was observed in literature (Longstaff \& Heath, 2006; Poluha \& Teulings, 1998; Mc Lennan et al., 1972).

Levodopa treatment appeared to positively influence the dimension of the spiral in the $67.7 \%$ of the subjects, who increased dimensions in the on condition with respect to the off condition, and approached control values. The poor effect of levodopa on subject PD_5 is noticeable from both the dimension parameter in the off and on condition and the difference between UPDRS OFF and UPDRSON. While the effect of levodopa on micrographia is not clearly explained by literature, it is well known that the treatment displays a positive effect on bradykinesia, thus increasing the velocity of motion and the motor control capabilities of the subjects when passing from the off to the on medication condition.

Velocity profiles analysis of hand-drawn spirals showed that $\mathrm{H}$ subjects increased their velocity from the centre to the outside of the spiral (Pullman, 1998); for PD subjects the analysis of velocity profiles showed a higher difficulty in maintaining constant increments between spires, with a major slowness of the movement. As a result, the ranges of velocity and the angular coefficient of linear regression, (which represent the capacity to increment the velocity from the inside to the outside of the spiral and the extent of the increment) were smaller for PD subjects, and in particular the lowest values were registered in the off medication condition, were the absence of levodopa contributed to worsening bradykinesia. As a conclusion, pathological subjects are affected by bradykinesia, fatigue and a general loss of motor control, which cause slower drawing, reduced drawing dimensions and loss of accuracy.

\subsection{Clock Drawing Test}

In this study an experimental set-up was defined for the quantitative and objective assessment of cognitive function through the analysis of drawing. This set up was firstly applied to a group of healthy subjects (CG) and then to a group of subjects with Parkinson's Disease (PD) and to a group of subjects with Parkinson's Disease and dementia (PDD), to assess the validity of the set-up in the clinical usage.The subjects were administered the Clock Drawing Test (CDT), a common 
clinical test for the assessment of cognitive dysfunction. The test was administered in two different modalities: the command and the copy condition. Each of these investigates specific cerebral areas and thus is sensitive to differently located brain damages (Shah, 2002).

Rather than concentrating on the CDT scoring itself, our aim was to develop a quantitative measurement. The CDT, in fact, has always been visually rated by the clinician, while only a few attempts were made to introduce objective and quantitative parameters (Chiu \& Li, 2008). We analyzed duration of the trials (Trial Duration, TD) for the two conditions of test. We found, indeed, that duration of the trial can be a useful, objective parameter for the assessment of the cognitive performance.

The calculation of correlation between MMSE scores and duration of the trials revealed that a higher degree of dementia led to longer durations of the trials, presumably because the impaired subjects had more reasoning difficulties.

In both motor and cognitive drawing tests the graphic gesture was acquired with an optoelectronic system with six cameras. Markers were put on the pen and on the sheet. All trials revealed to be well tolerated by the subjects, who were able to complete the requested tasks without excessive fatigue.

The optoelectronic system revealed to be advantageous both for a correct measurement of the parameters and for being a practical, non-invasive technique. In fact it allowed the subject to freely draw, recreating the "pen and sheet" condition and thus allowing more natural movements.

The computation of the parameters of interest was carried out using Smart Analyzer software and, starting from the acquisition of the gesture with the optoelectronic system, it allows everyone to compute the data automatically, using the related protocol.

The protocol allows an easy employment in clinic analysis, thanks to the simple movements requested, the little duration, the complete non invasivity and the natural conditions of testing.

This study has some limitation. First of all the small number of participants, in particular for the assessment of cognitive capabilities - clock drawing test (CDT), resulted in limited strength of the clinical and statistical findings. Then this experimental set-up takes into account the graphic gesture and does not evaluate the whole body motion during the test; however it is the first attempt to quantify this gesture using optoelectronic systems and with an appropriate integration of signals, it is in addition possible to analyse muscle activation (using electromyography) during the graphic gesture execution.

\section{Further research}

The instrumentation is suitable for a further development of drawing and writing tests; these characteristics suggest the applicability of these protocols with regard not only to PD but also to other fields of motor and cognitive evaluation, both in the preand post-medication phases.

Besides, the protocol could be employed for the quantitative characterization of other graphic movements (including writing), particularly suitable for clinical applications. The combination of the graphic tests and the optoelectronic instrumentation can in 
fact start a brand new strand of studies about the 3D measurement of the graphic gesture.

We are actually developing a new protocol for the evaluation of developmental problems (dyslexia, dysgraphia) in children, based on the administration of graphic tests (Denver Developmental Screening Test) and on the assessment of both the graphic trace and the body position during writing and drawing.

We found, in fact, that body position represents important additional information when assessing graphic capabilities: for instance, the head position influences the sight area, while the superior limbs position influences the drawing itself.

In this case the positioning of the markers on the pen and sheet remains the same of the protocol described hereby, while some additional markers are put on the superior limbs, trunk and head (Figure 7).

With an appropriate integration of signals, which is supported by the optoelectronic system, it is in addition possible to analyse muscle activation (using integrated electromyography) during the graphic gesture execution.

Our protocol has been therefore enriched by the application of superficial electrodes on the biceps brachii, triceps brachii, brachioradialis and flexorcarpumulnaris muscles.

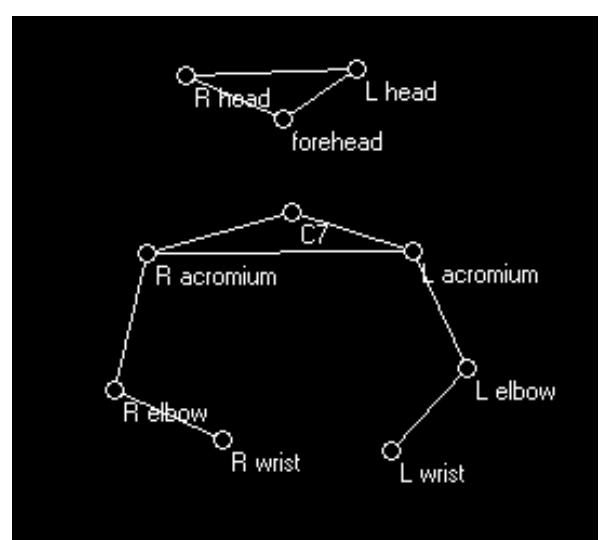

Fig. 7. Marker positioning on the subject for the assessment of body position during graphic tests

The optoelectronic systems are already present in hospitals and clinics that are concerned with PD and are up-to-date with Gait Analysis; the introduction of graphic tests will thus enhance the possibilities of clinical evaluation and screening. Finally it must be underlined that, unlike many other tests, these tests are completely not invasive and easily administrable, suitable for infants, elderly subjects or subjects with dementia, a point that is of great importance in reducing the patients' annoyance.

\section{References}

Balàs I, Llumiguano C \& Dòczi TP. Ablative stereotactic surgery improves manual performance time in Parkinson's disease. Parkinsonism Relat Disord.,12(4), May 2006, 223-7, ISSN: 1353-8020

Caligiuri MP, Teulings HL \& Filoteo JV. Quantitative measurement of handwriting in the assessment of drug-induced parkinsonism. Hum Mov Sci., 25(4-5), May 2006, 510-22, ISSN: 0167-9457 
Vimercati, S.; Galli, M.; De Pandis, M. F.; Cimolin, V. \& Albertini, G.: Evaluation...

Chiu YC \& Li CL. Sensitivity and specificity of the Clock Drawing Test, incorporating Rouleau scoring system, as a screening instrument for questionable and mild dementia: scale development. Int J Nurs Stud., 45(1), Jan 2008, 75-84, ISSN:0020-7489

Emre M \& Aarsland D. Clinical diagnostic criteria for dementia associated with Parkinson's Disease. Mov Disord, 22(12), Sept 2007, 1689-707, ISSN: 15318257

Goetz C \& Emre M. Parkinson's Disease dementia: definitions, guidelines and research perspectives in diagnosis. Ann Neurol., 64, Dec 2008, (suppl):S81S92, ISSN: 1531-8249

Hubbard EJ \& Santini V.Clock drawing performance in cognitively normal elderly. Arch Clin Neuropsychol., 23(3), May 2008, 295-327, ISSN1873-5843

Lange KW, Mecklinger L \& Walitza S. Brain dopamine and kinematics of graphomotor functions. Hum Mov Sci., 25(4-5), Jul 2006, 492-509, ISSN: 0167-9457

Longstaff MG \& Heath RA. Spiral drawing performance as an indicator of fine motor function in people with multiple sclerosis. Hum Mov Sci., 25(4-5), Oct 2006, 474-91, ISSN: 0167-9457

Longstaff MG, Mahant PR \& Satcy MA. Discrete and dynamic scaling of the size of continuous graphic movements of parkinsonian patients and elderly controls. $J$ Neurol Neurosurg Psychiatry., 74(3), Mar 2003, 299-304, ISSN: 00223050

McLennan JE \& Nakano K. Micrographia in Parkinson's disease. Journal of the Neurological Sciences, 15 (2), Feb 1972, 141-152, ISSN: 0022-510X

Ondo WG, Wang A \& Thomas M. Evaluating factors that can influence spirography ratings in patients with essential tremor. Parkinsonism Relat Disord., 11(1), Jan 2005, 45-8, ISSN: $1353-8020$

Poluha P \& Teulings HL. Handwriting and speech changes across the levodopa cycle in Parkinson's disease. Acta Psychologica, 100 (1-2), Nov 1998, 71-84, ISSN: 0001-6918

Pullman SL. Spiral analysis: a new technique for measuring tremor with a digitizing tablet. Mov Disord.,13, 2008, Suppl 3:85-9, ISSN: 1531-8257

Riedel O \& Klotsche J. Cognitive impairment in 873 patients with idiopathic Parkinson's disease. J Neurol, 255 (2), Feb 2008, 255-264, ISSN: 1432-1459

Saunders-Pullman R, Derby C \& Stanley K. Validity of spiral analysis in Early Parkinson's Disease. Mov Disord.; 23(4), Mar 2008, 531-7, ISSN: 1531-8257

Seber SL, Kumar R \& Woo MA. Cognitive test performance and brain pathology. Nurs Res, 57(2), Mar-Apr 2008, 75-83, ISSN:00296562

Shah J. Only time will tell: Clock Drawing as an early indicator of neurological dysfunction. P\&S Medical Review. 7 (2), Spring 2001, 30-34

Stanley K \& Hagenah J. Digitized spiral analysis is a promising early motor marker for Parkinson Disease. Parkinsonism Relat Disord.,16(3), Mar 2010, 233-4, ISSN: 1353-8020

Tucha O, Mecklinger L \& Thome J. Kinematic analysis of dopaminergic effects on skilled handwriting movements in Parkinson's disease. J Neural Transm., 113(5), May 2006, 609-23, ISSN1435-1463

***http://cpmcnet.columbia.edu/news/review/pdf/p30-34.pdf 